\title{
GENERALIZED QUOTIENT RINGS
}

\section{FRED RICHMAN ${ }^{1}$}

1. Introduction. If $A$ is an integral domain and $S$ is a subset of $A$ not containing zero, then one can form the quotient ring $A_{S}$ which lies between $A$ and its quotient field. It is well known that $A_{S}$ is flat as an $A$-module. The motivation for this paper is to determine how much the converse of this theorem holds. The objects of study are therefore rings $B$ between $A$ and its quotient field which are flat as $A$-modules. We obtain two characterizations of these "generalized quotient rings" and show that they enjoy many of the properties of ordinary quotient rings. Extensions related to quotient rings in the same manner that invertible ideals are related to principal ideals are also examined. A subsidiary result is a new characterization of Prüfer rings.

The properties of flatness used in the sequel can be found in [1] or in the exercises of [2]. For standard results in commutative rings the reader is referred to [3] and [4].

All rings will be integral domains. An extension $B$ of a ring $A$ will mean an extension in the quotient field of $A$ and will of ten simply be indicated by the notation $A \subset B$. If $A \subset B$ and $I$ is an ideal of $A, J$ an ideal of $B$, then $I^{\theta}$ will stand for the ideal in $B$ generated by $I$, i.e., $I B ; J^{c}$ will stand for the ideal $J \cap A$ of $A$. Where applicable, $Y:{ }_{z} X$ stands for $\left\{\left.z \in Z\right|_{z X} \subset Z Y\right\}$ with the subscript $Z$ omitted when obvious.

2. Characterizations. A natural point of departure is the study of prime ideals. For the extension $A \subset A_{S}$ every prime ideal $P$ of $A$ has the property that either $P^{\boldsymbol{b}}=A_{S}$ or $A_{S} \subset A_{P}$. Dubbing this condition on an extension good, we put it into more workable form.

Lemma 1. $A \subset B$ is good if and only if $(y: x) B=B$ for all $x / y \in B$.

Proof. Notice that if $P$ is a prime ideal of $A$, then $B \nsubseteq A_{P}$ if and only if $(y: x) \subset P$ for some $x / y \in B$. This, combined with the fact that $(y: x) B \neq B$ if and only if for some prime ideal $P \supset(y: x), P B \neq B$, yields the lemma.

REMARK. The observation that if $\left\{I_{j}\right\}$ is a finite set of ideals of $A$ such that $I_{j} B=B$ for all $j$, then $\left(\cap I_{j}\right) B=B$ can be used to strengthen the right-hand condition of the lemma. 1964.

Presented to the Society, January 26, 1964; received by the editors March 30 ,

${ }^{1}$ This is part of a Ph.D. dissertation written at the University of Chicago. 
This condition characterizes generalized quotient rings.

Theorem 1. Let $A$ be an integral domain, $B$ a subalgebra of the quotient field of $A . A \subset B$ is good if and only if $B$ is a flat $A$-module.

Proof. To show $B$ is flat it suffices to show that the natural map $H \otimes_{A} B \rightarrow B$ is 1-1 for any ideal $H$ of $A$. Suppose $c=\sum h_{j} \otimes b_{j} \rightarrow 0$, $h_{j} \in H, b_{j} \in B$, i.e., $\sum h_{j} b_{j}=0$. Let $I=\bigcap\left(A:{ }_{A} b_{j}\right)$. Clearly $I c=0$. But by Lemma 1 and the remark, $I B=B$. Therefore $I c=0=B 0=B I c$ $=B c$ and so $c=0$.

Suppose $B$ is flat. If $x / y \in B$, then $y \cdot x / y-x \cdot 1=0$. Therefore there exist $a_{i j} \in A$ and $b_{j} \in B$ such that

$1^{\circ} x / y=\sum a_{1 j} b_{j}$,

$2^{\circ} 1=\sum a_{2 j} b_{j}$,

$3^{\circ} y a_{1 j}-x a_{2 j}=0$.

If $P$ is a prime ideal of $A$, then either $a_{2 j} \in P$ for all $j$, in which case by $2^{\circ} P B=B$, or for some $j, a_{2 j} \notin P$, in which case by $3^{\circ} x / y=a_{1 j} / a_{2 j}$ which is in $A_{P}$.

Another characterization of generalized quotient rings shows that they are determined by local properties. First we need a useful lemma.

Lemma 2. If $A \subset B \subset C$, then the following two implications hold.

(1) If $A \subset C$ is flat, then $B \subset C$ is flat.

(2) If $A \subset B$ is flat and $B \subset C$ is flat, then $A \subset C$ is flat.

Proof. To prove (1) we note that if $x / y \in C$, then $\left(y:{ }_{A} x\right) C=C$ and so," a fortiori, $\left(y:_{B} x\right) C=C$. (2) is a well-known property of flatness.

Theorem 2. Let $A$ be an integral domain, $B$ a subalgebra of the quotient field of $A . A \subset B$ is flat if and only if $B_{M}=A_{M \cap A}$ for all maximal ideals $M$ of $B$.

Proof. If $A \subset B$ is flat, then $A \subset B_{M}$ is flat and so $A_{M \cap A} \subset B_{M}$ is flat by Lemma 2. Suppose $x / y \in B_{M}, x, y \in A_{M \cap A}$. Then $(y: x) B_{M}$ $=B_{M}$ and so $(y: x)=A_{M \cap A}$. Therefore $x=a y, a \in A_{M \cap A}$, and so $x / y \in A_{M \cap A}$.

Suppose there exists $x / y \in B$ such that $(y: x) B \neq B$. Let $M \supset(y: x) B$, $M$ a maximal ideal of $B$. Clearly $(y: x) B_{M} \neq B_{M}$ and so $A \subset B_{M}$ is not flat and thus $B_{M} \neq A_{M \cap A}$.

Notice that the theorem and proof remain valid if the word "maximal" is replaced by the word "prime" throughout.

COROLlaRy. Every flat extension is an intersection of localizations, i.e. if $A \subset B$ is flat, then $B=\cap A_{P}$ where $P$ runs over some set of prime ideals of $A$. 
The converse is false.

3. Extension and contraction of ideals. We now show that finite intersections of primary ideals "behave right" under extension and contraction and thus get a complete picture in the Noetherian case. First a preliminary lemma.

Lemma 3. If $A \subset B$ is flat, $\left\{Q_{j}\right\}$ is a finite set of primary ideals such that $Q_{j}^{e} \neq B, P_{j}=\operatorname{Rad}\left(Q_{j}\right)$ and $I=\cap Q_{j}$, then $P_{j}^{e} \neq B$ and $I^{e}=I_{S} \cap B$, where $S=A-\bigcup P_{j}$.

Proof. Clearly $P_{j}^{e} \neq B$. Since $A \subset B$ is flat, we have $B \subset A_{S}=\cap A_{\boldsymbol{P}_{j}}$. Suppose $t / s \in I_{S} \cap B$, i.e. $t \in I, s \in S$ and $t / s \in B$. By the flatness of $B$ there exist $a_{i k} \in A$ and $b_{k} \in B$ such that

$1^{\circ} t / s=\sum a_{1 k} b_{k}$,

$2^{\circ} 1=\sum a_{2 k} b_{k}$,

$3^{\circ} s a_{1 k}-t a_{2 k}=0$.

By $3^{\circ} a_{1 k} \in Q_{j}$ and so by $1^{\circ} t / s \in I^{e}$. Clearly $I^{e} \subset I_{S} \cap B$.

Theorem 3. Let $A$ be an integral domain, $B$ a generalized quotient ring of $A, \mathfrak{B}$ the set of ideals of $B$ admitting a finite primary representation, $\mathfrak{A}$ the set of ideals of $A$ admitting a finite primary representation $\cap Q_{j}, Q_{j}^{j} \neq B$. Then $e$ and $c$ give a 1-1 correspondence between $\mathfrak{A}$ and $\mathfrak{B}$ preserving prime ideals, primary ideals and finite intersections. Furthermore, if $I$ is an ideal of $A$ admitting a finite primary representation $\cap Q_{j}, 1 \leqq j \leqq n$, where $Q_{j}^{e} \neq B$ for $1 \leqq j \leqq k$ and $Q_{j}^{e}=B$ for $k<j \leqq n$, then $I^{e c}=\cap Q_{j}, 1 \leqq j \leqq k$.

Proof. Clearly $c$ maps $\mathfrak{B}$ into $\mathfrak{A}$. Consider $B \subset A_{S}=\bigcap_{P_{j}}$ where $P_{j}$ runs over the radicals of the (finitely many) primary ideals in question. Then $I^{e}=I_{S} \cap B$ for all ideals $I$ in question (Lemma 3). Therefore $e$ is the composite of extension into a quotient ring and contraction, both of which (since $S$ is prime to all ideals in question) preserve prime ideals, primary ideals and finite intesections. If $I$ is an ideal in $\mathfrak{A}$, then $I^{e c}=\left(I_{S} \cap B\right) \cap A=I_{S} \cap A=I$ since $S$ is prime to $I$. If $J$ is an ideal in $\mathfrak{B}$, then $J^{c e} \subset J$. If $t / s \in J$, then $t \in J^{c}$. But $J^{c e}=\left(J^{c}\right)_{S} \cap B$ and so $t / s \in J^{c c}$. Finally, if $I$ is an ideal in $\mathfrak{A}$ and $K$ is an ideal of $A$ such that $K^{e}=B$, then $(I \cap K)^{e c}=I$. Indeed,

$$
I=I^{e c} \supset(I \cap K)^{e c} \supset(I K)^{e c}=(I K B) \cap A=I B \cap A=I^{e c}=I .
$$

Recalling Cohen's Theorem that a ring is Noetherian if every prime ideal is finitely generated, we have as an immediate consequence:

Corollary. If $A$ is Noetherian and $A \subset B$ is flat, then $B$ is Noetherian, every ideal of $B$ is extended and an ideal $I$ of $A$ is contracted if and only if no prime ideal belonging to $I$ blows up in $B$. 
4. Integral dependence. Generalized quotient rings also respect integral closure.

Proposition 1. Let $A$ be an integral domain, $B$ a generalized quotient ring of $A$. Then $B^{*}=A^{*} B$ is a generalized quotient ring of $A^{*}$, where $X^{*}$ is the integral closure of $X$.

Proof. That $B^{*} \supset A^{*} B$ is trivial. Suppose $x \in B^{*}$, i.e. there exist $b_{j} \in B$ and a positive integer $n$ such that $x^{n}+b_{n-1} x^{n-1}+\cdots+b_{0}=0$. Let $I=\cap A:{ }_{A} b_{j}$. Then $I x \subset A^{*}$ and $I B=B$. Therefore $x B=x I B \subset A^{*} B$ and so $x \in A^{*} B$. Moreover, since $I C A^{*}:{ }_{A^{*} X}$ and $I B=B,\left(A^{*}:{ }_{A^{*}} x\right) B^{*}$ $=B^{*}$.

Corollary. If $A \subset B$ is flat and $A$ is integrally closed, then $B$ is integrally closed.

We note the following relationship between generalized quotient rings and integral dependence.

Proposition 2. If $A \subset B$ is flat and $B$ is integral over $A$, then $A=B$.

Proof. If $x / y \in B$, then $(y: x) B=B$ and so $(y: x)=A$ since no proper ideal blows up in an integral extension. Therefore $x / y \in A$.

5. Invertible extensions. Quotient rings are generated by elements of the form $1 / s$. In general, an extension generated by elements of the form $x / y$ where the ideal $(x, y)$ is principal is a quotient ring. The usual generalization of principal to invertible leads one to consider invertible extensions $A \subset B$ where $B$ is generated by elements $x_{j} / y_{j}$, $\left(x_{j}, y_{i}\right)$ an invertible ideal of $A$. Clearly every quotient ring is invertible and an invertible extension, being a direct limit of projective modules, is flat. In particular, any extension of a Dedekind domain is flat. By choosing $A$ to be a Dedekind domain with nontorsion class group, we can get a nontrivial example of a generalized quotient ring. Indeed, let $M$ be a maximal ideal of $A$ such that $M^{n}$ is not contained in a principal ideal for any positive integer $n$ and let $B$ be generated by $M^{-1}$. If $1 / s \in B$ for $s \in A$, then $1 \in s M^{-n}$ for some positive integer $n$ and so $M^{n} C(s)$. Therefore $(s)=A$. Thus $B$ contains no nontrivial reciprocal of an element of $A$ and so cannot be a quotient ring. These are essentially the only examples we have.

It is unlikely that every flat extension is invertible. In the following special case, however, we have equivalence.

Proposition 3. Let $A$ be an integral domain, $x / y$ an element of the quotient field of $A$ and $B=A[x / y]$. If $B$ is flat and $A$ is integrally closed then the ideal $(x, y)$ is invertible. 
Proof. Since $A \subset B$ is flat, we have that $(y: x) B=B$, i.e. there exist $h_{j} \in(y: x)$ such that $h_{n} x^{n} / y^{n}+h_{n-1} x^{n-1} / y^{n-1}+\cdots+h_{1} x / y+h_{0}=1$, for some non-negative integer $n$. Multiplying by $y^{n} / x^{n}$, we get that $y / x$ is integral over $A_{S}, S=\left\{\left(h_{0}-1\right)\right\}$, and so $y / x \in A_{S}$ since $A_{S}$ is integrally closed. Therefore $y / x=h /\left(h_{0}-1\right)^{t}$ for some $h \in A$ and some non-negative integer $t$, and so $h x / y+a h_{0}=1$ for some $a \in A$, i.e. $(h / y) x+\left(a h_{0} / y\right) y=1$, where $h / y$ and $a h_{0} / y \in(x, y)^{-1}$.

If $A$ is a unique factorization domain or has only finitely many prime ideals, it is easily seen that any intersection of localizations is a quotient ring. In particular, for this class of rings, every generalized quotient ring is a quotient ring (and therefore invertible).

The most hopeful way of relating arbitrary flat extensions to invertible extensions appears to be the following conjecture: If $A \subset B$ is flat and $A \neq B$, then there exists an element $x / y$ in $B$ but not in $A$ such that the ideal $(x, y)$ is invertible. If this were true, then any flat extension could be realized by a transfinite sequence of invertible extensions. Even when $A$ is quasi-local and invertible reduces to principal, this question does not seem to be easily settled. It is false for arbitrary intersections of localizations of a local ring.

6. Prüfer rings. If $A$ is a Prüfer ring, then any extension of $A$ is invertible and hence flat. We shall show this to be a characteristic property of Prüfer rings.

Lemma 4. Let $R$ be a ring with exactly one maximal ideal $M$ such that every extension of $R$ is flat. Then $R$ is a valuation ring.

Proof. Suppose $x / y \notin R, x, y \in R$. Then $(y: x) \subset M$. Since $R[x / y]$ is flat, $(y: x)$ blows up in $R[x / y]$. Therefore there exist $m_{j} \in M$ such that $m_{0}+m_{1} x / y+\cdots+m_{n} x^{n} / y^{n}=1$, for some positive integer $n$. Multiplying by $y^{n} / x^{n}$, we get that $y / x$ is integral over $R$. But $R[y / x]$ is flat and so by Proposition $2, y / x \in R$.

THEOREM 4. If $A$ is an integral domain such that every extension of $A$ into its quotient field is flat, then $A$ is a Prïfer ring.

Proof. If $B$ is an extension of $A_{M}$ for some maximal ideal $M$ of $A$, then $A \subset B$ is flat and therefore by Lemma $2, A_{M} \subset B$ is flat.Therefore $A_{M}$ is a valuation ring for any maximal ideal $M$ of $A$ by Lemma 4 and so $A$ is a Prüfer ring.

We note that this theorem says that the weak global dimension of a ring is no greater than one if every extension has weak dimension zero and pose the question of whether this result can be extended to higher dimensions. 


\section{REFERENCES}

1. N. Bourbaki, Algèbre commutative, Hermann, Paris, 1961.

2. H. Cartan and S. Eilenberg, Homological algebra, Princeton Univ. Press, Princeton, N. J., 1956.

3. M. Nagata, Local rings, Interscience, New York, 1962.

4. O. Zariski and P. Samuel, Commutative algebra, Vols. 1, 2, Van Nostrand, New York, 1958-1960.

New Mexico State University

\section{ON ASSOCIATIVE DIVISION ALGEBRAS OF PRIME DEGREE ${ }^{1}$}

\section{A. A. ALBERT}

In 1938 Richard Brauer showed ${ }^{2}$ that, if $\mathfrak{D}$ is an associative division algebra of degree five over its center $\mathfrak{F}$, there is a field $\Re$ of degree at most twelve over $\mathfrak{F}$ such that the scalar extension $\mathfrak{D} \times \Omega$ is a cyclic algebra over $\Omega$. Indeed $\Omega=\mathfrak{F}\left(\alpha_{1}, \alpha_{2}, \alpha_{3}\right)$, where $\alpha_{1}$ is a root of a quadratic equation over $\mathfrak{F}, \alpha_{2}$ is a root of a quadratic equation over $\mathfrak{F}\left(\alpha_{1}\right)$, and $\alpha_{3}$ is a root of a cubic equation over $\mathfrak{F}\left(\alpha_{1}, \alpha_{2}\right)$. Since that time there has been no progress in the study of the structure of associative central division algebras of prime degree.

In view of Brauer's result it seems reasonable, as a first step in the study of central division algebras $\mathfrak{D}$ of prime degree $p$ over $\mathfrak{F}$, to consider the case where there is a quadratic field $\Omega$ over $\mathfrak{F}$ such that $\mathfrak{D}_{\Omega}$ is cyclic. Then $\mathfrak{D}_{\Omega}=\mathfrak{D} \times \Omega$ has a subfield $\mathbb{B}$ which is cyclic of degree $p$ over $\Omega$. The simplest subcase is that where $B$ is actually normal, but, of course, not cyclic over $\mathfrak{F}$. We shall treat this case under the assumption that $\mathfrak{F}$ has characteristic $p$, and shall prove that then $\mathfrak{D}$ is a cyclic algebra over $\mathfrak{F}$.

Our proof proceeds as follows. We are assuming that $\mathfrak{D}$ is a central division algebra of prime degree $p$ over $\mathfrak{F}$ and that $\Re=\mathfrak{F}(t)$ is a quadratic extension of $\mathfrak{F}$. Let $J$ be the automorphism of $\Omega$ such that $t J=-t$. Then we may extend $J$ to an automorphism $J$ of $\mathfrak{D} \times \Re$ such that $d J=d$ for every $d$ in $\mathfrak{D}$. We are also assuming that there is an element $z$ in $\mathfrak{D} \times \Re$ such that the field $\mathbb{Z}=\Re(z)$ is not only cyclic over

Received by the editors April 1, 1964.

${ }_{1}$ This paper was sponsored in part by the National Science Foundation under NSF Grant GP 2424.

2 Proc. Nat. Acad. Sci. U. S. A. 24 (1938), 243-246. 